werden entscheidende Schritte in die Zukunft sein. Auf nationaler Ebene wird die Integration der Leitidee Nachhaltige Entwicklung in alle Politikbereiche erforderlich sowie die Ausgestaltung neuer Generationenverträge (Jörg Tremmel). Aktive Nachhaltigkeitsstrategien könnten dann auch zu neuen Arbeitsplätzen führen.

In der abschließenden Podiumsdiskussion wurde darüber diskutiert, welche Lösungsansätze zu einer "Nachhaltigen Informationsgesellschaft" führen können. Was könnten sozusagen Modelle und Strukturen einer informationstechnischen globalen civil society werden, eines sozusagen "vernetzten Superorganismus"? Denn bald werden schon 60 Millionen Menschen weltweit vernetzt sein. Gleichzeitig beginnen immer mehr Menschen ressourcenintensiv zu leben. Bereits heute werden $30 \mathrm{Mrd}$. bis 200 Mrd. Dollar über das Netz verdient. Die Informationsgesellschaft wird $\mathrm{zu}$ einer Beschleunigungsgesellschaft par exellence; sie baut auf eine Verbindung aus tradiertem Wissen und sich fortlaufend steigender Computerpower, so lautete ein Resumee der Tagung.

Um eine Nachhaltige Entwicklung zu erreichen, muß zunächst der Versuch im Vordergrund stehen, noch viel mehr die damit verbundenen komplexen Zusammenhänge $\mathrm{zu}$ verstehen. Klare Zielvereinbarungen müssen baldmöglichst aufgestellt werden, um einen schonenderen Umgang mit den Umweltressourcen, aber auch der Ressource Mensch zu erreichen. Einen breiten Beteiligungs- und Bildungsprozeß hierfür zu initieren, ermöglicht sowohl Manipulation durch die Informationstechnik zu verringern, als auch die Idee der Nachhaltigkeit im Bewußtsein breiter Bevölkerungsschichten $\mathrm{zu}$ verankern. Besonders gebraucht werden in Zukunft, außer technischen Innovationen, auch soziale und institutionelle, um langfristige "gesellschaftliche Pleiten" zu vermeiden, so ein weiteres Abschlußstatement der Tagung.

Insgesamt war die Konferenz ein großer Erfolg, wenn sie auch weniger dem Austausch eines Fachpublikums, sondern mehr einer breit angelegten Pressekonferenz gleichkam. Doch gerade darin zeigte sich, daß die Leitidee einer Nachhaltigen Entwicklung inzwischen schon auf breiter Front Fuß gefaßt hat und der Operatinalisierung dieser Idee eigentlich nichts mehr im Wege stehen sollte.
* Forschungsinstitut für anwendungsorientierte Wissensverarbeitung (FAW) (Hrsg.): Herausforderung 2025 - Auf dem Weg in eine weltweite nachhaltige Informationsgesellschaft. Ulm, Juni 1998. Zu beziehen über FAW, Fax: ++ 49 (0) 731/501-111; oder Forum Info 2000, Fax: ++ 49 (0) 228/98538-22

\section{Verkehrswissenschaftliche Tage in Dresden}

\section{Tagungsbericht von Daniela Georgiewa, ITAS}

In der Zeit vom 4. bis 5. Juni 1998 fanden die 17. Verkehrswissenschaftlichen Tage in Dresden unter der Schirmherrschaft des Bundesministers für Bildung, Wissenschaft, Forschung und Technologie, Dr. Jürgen Rüttgers, statt. Damit greift die Fakultät Verkehrswissenschaften "Friedrich List" der Technischen Universität Dresden die im November 1954 begründete Tradition dieser renommierten wissenschaftlichen Konferenz wieder auf, die bis 1989 alle vier Jahre stattfand. Die Verkehrswissenschaftlichen Tage in Dresden stellten und stellen ein Forum für den internationalen Meinungs- und Erfahrungsaustausch zu wissenschaftlichen Fragen des Verkehrs- und Nachrichtenwesens sowie der Telekommunikation dar. Neben der Budapester Verkehrskonferenz boten sie bis zur Wende die wichtigsten Austauschmöglichkeiten zwischen Verkehrswissenschaftlern aus Ost und West.

Trotz einer geringeren Beteiligung von Vertretern osteuropäischer Länder stieß auch die diesjährige Dresdner Tagung auf ein großes Interesse mit insgesamt rd. 450 Teilnehmern. Sie hatte den Titel "Integrierte Mobilitätsforschung - Herausforderungen für die interdisziplinäre Zusammenarbeit" und behandelte in einer Plenarveranstaltung sowie in fünf parallel durchgeführten Tagungssektionen neben den herkömmlichen verkehrswissenschaftlichen Themenbereichen Ökonomie, Fahrzeugtechnik und Planung auch Fragen der verkehrsbezogenen Information und Kommunikation (Telematik) sowie der nachhaltig umweltverträglichen Entwicklung des Verkehrs. 
Insgesamt wurden rund 60 Vorträge gehalten, an die sich zum Teil eine lebhafte, aus Zeitgründen aber nicht immer sehr ausführliche Diskussion anschloß. Hierfür ergaben sich jedoch vielfältige Gelegenheiten am Rande der Tagung.

Vor dem Gesamtplenum sprachen Vertreter des Bundesministeriums für Bildung, Wissenschaft, Forschung und Technologie, des Bundesministeriums für Verkehr sowie der Organisation for Economic Cooperation and Development (OECD). Sie boten einen informativen Überblick über neuere Forschungs- und Förderansätze zur Mobilität, die deutsche Verkehrspolitik vor dem Hintergrund des europäischen Einigungsprozesses sowie aktuelle internationale Forschungsaktivitäten zur nachhaltig umweltverträglichen Verkehrsentwicklung.

Die Vorträge in den fünf Tagungssektionen lagen überwiegend bereits zur Tagung in ausführlicher Fassung vor. Sie sind in fünf Tagungsbänden dokumentiert (leider ohne durchgängige Pagination) und können vom Veranstalter bezogen werden.

Alle Sektionen enthalten Vorträge zu Themen, die die verkehrswissenschaftliche Forschung nicht nur heute, sondern auch längerfristig beschäftigen dürften. Von besonderem Interesse sind hier die zahlreichen Berichte über laufende oder abgeschlossene Forschungsprojekte, die zum Teil auch schon in die Praxis umgesetzt sind. Die Hauptbedeutung dieser Vorträge dürfte darin bestehen, daß sie zu einem verstärkten Überschreiten regionaler und nationaler Grenzen bei der grundlagen- und empirieorientierten Verkehrsforschung und zu einer Fortsetzung des intensiven Austauschs zwischen den vielfältigen Teildisziplinen der Verkehrsforschung auffordern.

Die jeweils einer Tagungssektion gewidmeten Tagungsbände: Wirtschaft und Verkehr (Band I); Verkehrstelematik und Betrieb (Band II); Entwicklungstendenzen der Fahrzeugtechnik (Band III); Verkehrsplanung, Verkehrstechnik und Anlagengestaltung (Band IV); Ökologische Nachhaltigkeit im Verkehr (Band V) sind erhältlich bei: Technische Universität Dresden, Fakultät Verkehrswissenschaften "Friedrich List" - Dekanat, Hettnerstr. 1-3, D-01069 Dresden.

\section{Politische Eliten in der Medien- gesellschaft} Notizen zu einer Tagung in Otzenhausen,
3. - 5.6.1998

von Bernd Wingert, ITAS

Das europäische Bildungszentrum Otzenhausen liegt in den hügeligen Ausläufern des Hunsrück, am Autobahndreieck bei Nonnweiler, und damit nicht weit von jenem Länderdreieck entfernt, wo sich Saarland, Luxenburg und Lothringen treffen. $\mathrm{Da} \beta$ es am Starnberger See, in Bad Boll oder in Bad Herrenalb Akademien gibt, ist dem wißbegierigen Tagungsbesucher hinlänglich bekannt. Aber Otzenhausen? Eine "Europäische Akademie" (die einer der Träger des Bildungszentrums ist) in eine solche ruhige Ecke zu legen, mag die heimliche Botschaft transportieren, daß der europäische Gedanke Ruhe braucht und ein wenig Luxus durchaus vertragen kann, um befördert zu werden; jedenfalls sind Service, Atmosphäre und Ausstattung des Zentrums exzellent und für Seminarplaner sicher eine gute Wahl.

Nunmehr zum dritten Mal hatten Peter Winterhoff-Spurk und Michael Jäckel zu einer Tagung eingeladen - der erste Professor für Medienpsychologie in Saarbrücken, der zweite Professor für Soziologie in Trier. Vor zwei Jahren ging es um die Frage einer sich eventuell herausbildenden "medialen Klassengesellschaft", dieses Mal um einen anderen Aspekt der Medien: "Politische Eliten in der Mediengesellschaft". Die Tagung lief über zweieinhalb Tage und war vom Thema her sorgfältig aufgebaut. Nach einer medienpsychologischen Einführung durch Winterhoff-Spurk und einer weiteren, nun kommunikationssoziologischen, durch Jäckel, gab es drei Themenblöcke. Sie waren Fragen der Rekrutierung der politischen Eliten, der Darstellung in den Medien und der Analyse von Medienwirkungen gewidmet. Jeder Block wurde zum einen eher psychologisch, zum anderen eher soziologisch angegangen. Den Abschluß bildete ein Beitrag von Dieter Zimmer (ZDF, Redaktionsleiter Dokumentation und Reportage) mit der Innenperspektive, "Politik im Fernsehen - von innen betrachtet".

Der thematische Fahrplan wurde mit zwei Zugaben versehen, einem Experiment und einer 